\title{
Aplicación del mainstreaming de género en las organizaciones a través de las certificaciones
}

\author{
Implementation of gender mainstreaming in organizations through \\ certifications
}

\author{
Concepción MIMBRERO MALLADO ${ }^{1}$ \\ Leonor María CANTERA ESPINOSA ${ }^{1}$ \\ Joilson Pereira da SILVA²
}

\begin{abstract}
Resumen
Las desigualdades entre mujeres y hombres se siguen manteniendo en las organizaciones a pesar de los esfuerzos políticos y sociales realizados para afrontar este fenómeno. Las certificaciones de igualdad son reconocidas en España como uno de los instrumentos políticos diseñados para combatir estos desequilibrios de género a partir de la promulgación de la Ley Orgánica n³/2007 para la igualdad efectiva de mujeres y hombres. Este artículo tiene como objetivo identificar el enfoque desde el que las organizaciones, públicas y privadas, responsables de la promoción de certificaciones de igualdad de género, están respondiendo a la aplicación del mainstreaming de género. Aplicamos un análisis de contenido a partir del estudio de publicaciones relativas a las certificaciones. Los resultados muestran que estas certificaciones se conceden, en muchos casos, ignorando indicadores esenciales para implementar el enfoque de género. Igualmente, se impulsa su uso lucrativo como marca de calidad organizativa.
\end{abstract}

Palabras clave: Certificación; Educación e igualdad; Género; Gestión de la calidad; Organizaciones.

\begin{abstract}
Inequalities between women and men persist in organizations in spite of political and social efforts to fight this phenomenon. Gender equality certifications have been recognized in Spain as one of the political measures aimed at combating gender imbalances since the promulgation of the organic Law $n^{\circ}$ 3/2007 on effective equality of women and men. This article aims to identify the approaches adopted by public and private organizations that are responsible to promote gender equality certifications in response to gender mainstreaming implementation. Content analysis of publications related to gender certifications was carried out. The results show that in many cases these certifications are granted disregarding essential indicators to implement the gender mainstreaming approach. Similarly, they have been used to boost profit since it indicates a distinguishing quality of these organizations.
\end{abstract}

Keywords: Certification; Educational equalization; Gender; Quality management; Organizations.

${ }^{1}$ Universidad Autónoma de Barcelona, Facultad de Psicología, Departamento de Psicología Social. Edificio B., Campus de Bellaterra, Código Postal 08193, Cerdanyola del Vallés, Barcelona, España. Dirección de correspondencia/Correspondence to: C. MIMBRERO MALLADO. E-mail: <conchi.mimbrero@gmail.com>.

2 Universidade Federal de Sergipe, Departamento de Letras-Libras, Programa de Pós-Graduação em Psicologia. São Cristóvão, SE, Brasil. 
El siglo $X X$ ha sido escenario de grandes transformaciones que afectan a las mujeres, logradas gracias a cambios socioeconómicos, políticos y culturales y sobre todo a las reivindicaciones del movimiento feminista y a la consecuente evolución en el campo de las políticas de igualdad de género. A nivel internacional, en la IV Conferencia Mundial de las Mujeres organizada por la Organización de las Naciones Unidas en Beijing (celebrada en Pekín en 1995), se adopta un nuevo enfoque: el mainstreaming o transversalidad de género y su reconocimiento dentro del ámbito internacional como estrategia fundamental para el logro de la igualdad entre mujeres y hombres. Así esta estrategia es entendida, según el Consejo de Europa (Instituto de la Mujer, 1999), como la reorganización, mejora, desarrollo y evaluación de procesos políticos, de forma que los actores involucrados habitualmente en la elaboración de las políticas adopten una perspectiva de igualdad entre los hombres y las mujeres en todos los ámbitos de acción, en todas las etapas y a todos los niveles. Este enfoque estratégico, se basa en las propuestas teóricas que cuestionan el carácter estructural del origen y mantenimiento de las desigualdades de género. En este artículo tomamos como referentes a Verloo y Roggeband (Roggeband \& Verloo, 2007; Verloo \& Roggeband, 1996) que manifiestan que los desequilibrios entre mujeres y hombres se deben a causas derivadas del orden social patriarcal, vinculadas a las estructuras básicas de la desigualdad y a sus mecanismos de reproducción.

En cuanto a las estructuras básicas de desigualdad, estas autoras reconocen que los desequilibrios entre mujeres y hombres se encuentran en la organización socioeconómica (fundamentalmente en la división sexual del trabajo) y en la organización de la vida privada (situándonos en los modos en los que organizamos la sociedad en términos de división sexual. Es decir, las normas y valores, instituciones y organización de cómo y con quién viven las personas, la reproducción y la sexualidad). Además en la organización sociopolítica referida al ejercicio de los derechos de ciudadanía (Verloo, 2001, 2005; Verloo \& Roggeband,
1996). En el caso de las mujeres, estas no suelen ser consideradas de la misma manera que los hombres, sino que más bien son relegadas, aún en la actualidad, a vivir una ciudadanía de segunda categoría (Brown, 2007; Reverter, 2011; Rodríguez, 2010).

En cuanto a los mecanismos básicos de reproducción de la desigualdad, engloban la distribución de recursos y la existencia de reglas formales e informales (Verloo \& Roggeband, 1996). La distribución de recursos públicos (materiales e inmateriales) así como el acceso a los mismos es diferente en hombres y en mujeres, siendo ellas las grandes damnificadas. Y las reglas formales o informales definen cómo son y cómo deben comportarse los géneros, salvaguardando los roles que definen la feminidad y la masculinidad.

En España, teniendo en cuenta el origen y mantenimiento de las desigualdades de género, en el ámbito organizacional (público y privado) surgen distintas herramientas para facilitar la aplicación del mainstreaming de género. Buen ejemplo de ello son los planes y las certificaciones de igualdad. Ambos, reconocidos como instrumentos políticos diseñados para combatir estos desequilibrios de género en las organizaciones a partir de la promulgación de la Ley Orgánica n³/2007 (España, 2007) para la igualdad efectiva de mujeres y hombres. Esta ley, prevé en su artículo 50 la creación de un distintivo empresarial en materia de igualdad para aquellas empresas que se destaquen por la aplicación de políticas de igualdad entre mujeres y hombres en las condiciones de trabajo, en los modelos de organización y en otros ámbitos, como los servicios, productos y publicidad de la organización.

A nivel privado surgen otras iniciativas, respondiendo al artículo 50 de la Ley Orgánica $n^{\circ}$ 3/2007 (España, 2007) para la igualdad efectiva de mujeres y hombres, como la diseñada por la Fundación Másfamilia: el modelo de certificación denominado efr1000 (empresa familiarmente responsable 1000).

En general, previamente al diseño de medidas para adoptar el enfoque de género que 
lleve al logro de las certificaciones, las organizaciones deben realizar una evaluación diagnóstica sobre la situación de hombres y mujeres que las constituyen (Biencinto \& González, 2010). No obstante, la realización de estos diagnósticos no es tarea fácil. Para ello, las organizaciones toman como referentes distintos modelos de aplicación del mainstreaming de género que se aplican a las políticas públicas y que coexisten actualmente en Europa, los denominados como modelos holandés, italiano, sueco, austriaco, irlandés, o alemán (Bustelo \& Lombardo, 2005, 2006; Mimbrero, 2014).

Este artículo formula la existencia de una relación entre los modelos de implementación del mainstreaming de género y los de aplicación de instrumentos políticos como las certificaciones de igualdad de género en las organizaciones. En concreto, y en el caso de España, Mimbrero (2014) apunta, que el gobierno central actual promueve como modelo de referencia el modelo alemán. En junio de 2002, la Federación de Municipios de Alemania incorporó el principio de transversalidad de género a su propio trabajo y actualmente esta estrategia se consolida a nivel local extendiéndose como práctica común a organizaciones de la sociedad civil tales como sindicatos y organizaciones privadas (Lombardo, 2005). Preocupada con la estandarización de los procesos organizacionales, Alemania vincula la implementación del mainstreaming de género con estándares de calidad (Mimbrero, 2014).

El marco anterior invita a que en el ámbito científico-académico-organizacional se formulen preguntas como: ¿cuáles son las organizaciones públicas y privadas responsables de la promoción de las certificaciones de igualdad de género?, ¿cómo las organizaciones públicas y privadas responsables de la promoción de las certificaciones de igualdad de género proyectan la aplicación del mainstreaming de género?, ¿desde qué posicionamiento se cuestionan las relaciones asimétricas ente mujeres y hombres para integrar el género en los procesos de certificación? y ¿qué metodología diagnóstica y de seguimiento se impulsan para implementar el género en los procesos de certificación y mantenimiento de las mismas?
Todo ello para conocer si en España, teniendo en cuenta la Ley Orgánica nº 3/2007 (España, 2007) para la igualdad efectiva de mujeres y hombres, se favorece o no la implementación del mainstreaming de género en las organizaciones. Para efecto de este artículo y englobando las preguntas anteriores, nos preguntamos de qué manera las organizaciones públicas y privadas en España (siguiendo la iniciativa de "Distintivo de Igualdad en las empresas") están respondiendo a la citada ley.

El objetivo general es pues, identificar el enfoque desde el que las organizaciones públicas y privadas (avaladas por el Gobierno de España) responsables de la promoción de las certificaciones de igualdad de género, están respondiendo a la aplicación del mainstreaming de género. A continuación pasamos a describir el método que guía este estudio; así como los resultados y la discusión de los mismos.

\section{Método}

Aplicamos un muestreo intencional de tipo opinático siguiendo un criterio estratégico. Este ha sido la búsqueda y selección de textos relativos a la certificación de la igualdad: normativos, técnicos de ayuda a organizaciones (manuales, guías e informes) y páginas webs. En concreto, analizamos el contenido de 35 publicaciones: 6 de carácter normativo, 14 páginas webs, y 15 documentos técnicos de ayuda a organizaciones para la implementación de las certificaciones y medidas de igualdad de género (manuales, guías e informes).

\section{Procedimientos}

Este artículo, acorde con los rasgos y principios propios de los estudios de género (Díaz \& Dema, 2013; Martínez et al., 2014), emplea un análisis de contenido desde un enfoque de metodología cualitativa. En concreto, nos apoyamos en la propuesta de Olabuénaga (2012) que desde el constructivismo sitúa la idea central 
del análisis de contenido en el texto original, que debe ser entendido y tratado como un escenario de observación del que se extrae información para someterla a un posterior análisis e interpretación.

Una vez seleccionados los textos, hemos seguido las fases de análisis de contenido del citado autor. Así, en un primer momento hemos elaborado un texto de campo a partir de la selección de datos o como señala Olabuénaga (2012) refiriéndose a los fragmentos de los textos respecto a los cuales indagamos sus temas, unidades de registro. Es decir, un documento en el que señalamos notas, aclaraciones y observaciones que nos han ayudado a describir la naturaleza de la realidad social que estudiamos.

A partir del texto de campo, elaboramos el texto de investigación. Este paso es crucial; porque como señala Olabuénaga (2012) el análisis de contenido toma una gran relevancia en un proceso de reducción de datos y formación de categorías (Olabuénaga, 2012).

Así, en este texto de investigación, partimos de las preguntas de investigación formuladas y teniendo en cuenta las notas y observaciones realizadas en el texto de campo advertimos una primera sistematización. Introducimos categorizaciones de la información extraída de los documentos analizados y las mismas fueron agrupadas en dos dimensiones: Dimensión 1 Enfoque organizativo. Esta dimensión integra las categorías que identifican las organizaciones promotoras de la certificación de la igualdad de género, los tipos de certificaciones y la perspectiva suscitada para su aplicación. Dimensión 2 Proyección del mainstreaming de género. Esta dimensión organiza las categorías referidas a la promoción del procedimiento de aplicación de la transversalidad de género en las organizaciones.

En cuanto a la categorización, además de lo anterior utilizamos lo que Olabuénaga (2012) define como categorías de tipo especiales. Siendo estas, para efecto de este estudio, aquellas que parten de indicadores diseñados para la aplicación del mainstreaming de género. Así, tomamos como referente el Protocolo para la implantación de un proceso de mainstreaming de género elaborado en el marco del Grupo de trabajo de mainstreaming de género, coordinado por el Instituto de la Mujer y la Unidad Administradora del Fondo Social Europeo (2013). Un documento que tiene entre sus objetivos el de proporcionar una herramienta con los pasos a seguir para implantar, en el seno de una organización, un proceso de mainstreaming de género. Además, el marco teórico sobre el origen y mantenimiento de las desigualdades de género de Roggeband y Verloo (2007) y Verloo y Roggeband (1996).

De acuerdo con el constructivismo, el texto de investigación fue sometido a juicio del equipo investigador para el análisis de la confiabilidad y autenticidad de las categorías, convirtiéndolo en un texto interpretativo en que representamos nuestra interpretación y versión científica de la realidad que estudiamos.

Así, en nuestro trabajo fueron extraídas dos grandes dimensiones citadas anteriormente que a su vez tienen sus respectivas categorías a saber:

Dimensión 1 - Enfoque organizativo: esta dimensión integra las siguientes categorías para el análisis de contenido de los textos seleccionados: (1) Organizaciones promotoras y distintivos: tipos de organizaciones responsables de la promoción de las certificaciones de igualdad de género y tipos de distintivos existentes. (2) Cultura organizativa: necesidad de una estructura organizativa para garantizar la articulación de la dimensión de género en los procesos de certificación. (3) Reconocimiento de las desigualdades de género y relaciones de poder: Conocimiento sobre el origen y mantenimiento de las desigualdades de género y el reconocimiento de relaciones asimétricas entre mujeres y hombres. (4) Interseccionalidad: la dimensión de género confluye con otras variables que también generan desigualdades.

Dimensión 2 - Proyección del mainstreaming de género: esta dimensión está constituida por tres categorías: (5) Marca de calidad: promoción de la certificación dentro de un sistema propio de 
gestión de calidad organizativa. (6) Transversalidad: adaptación del enfoque de género a todos los niveles y en todos los procesos de la organización. (7) Metodología: tipo de metodología propia de los estudios de género.

Una vez especificadas las categorías nos damos al análisis usando una doble estrategia, deductiva e inductiva (Berg, 1989; Olabuénaga, 2012; Pla, 1999). Por una parte, deductiva, asumiendo indicadores para la aplicación de la perspectiva de género en las organizaciones propuestos por el Instituto de la Mujer y la Unidad Administradora del Fondo Social Europeo (2013). En este caso, utilizando técnicas de análisis de reducción de datos que permiten inferir el contenido de los textos dentro de una u otra categoría establecida a priori. Por otra parte, inductiva, identificando en los documentos otras categorías relevantes extraídas del marco teórico sobre el origen estructural de las desigualdades entre mujeres y hombres que fundamenta la necesidad de la aplicación de la dimensión de género en las organizaciones (Verloo \& Roggeband, 1996). Un proceso en el que el equipo de investigación dirige la negociación que termina en consenso sobre las construcciones mejor argumentadas e informadas (Olabuénaga, 2012).

Cabe señalar que como parte del procedimiento se siguen las recomendaciones éticas, de acuerdo con lo previsto en las normativas que regulan este tipo de investigaciones, durante todo el proceso de estudio.

\section{Results}

Teniendo en cuenta las dimensiones encontradas y sus respectivas categorías, a continuación exponemos los resultados obtenidos:

\section{Dimensión 1: Enfoque organizativo}

1) Organizaciones promotoras y distintivos: en el análisis, hallamos dos tipos de organizaciones responsables de la promoción de las certificaciones de igualdad de género, públicas y privadas. Además, reconocemos diferentes distintivos de certificación en cada una de ellas.

Entre las instituciones públicas, encontramos el Instituto de la Mujer, adscrito al Ministerio de Igualdad, impulsador de las certificaciones de igualdad a partir de la promulgación de la Ley Orgánica n 3/2007, de 22 de marzo (España, 2007), para la igualdad efectiva de mujeres y hombres. En el año 2009, a nivel ministerial se aprueba el logotipo y representación gráfica del distintivo "Igualdad en la empresa" (España, 2009a) y se regula la concesión y utilización de este distintivo por el Real Decreto no 1615/2009, de 26 de octubre (España, 2009b). A partir del año 2014, mediante la Ley no 15/2014, de 16 de septiembre (España, 2014), se acuerda la integración de las competencias de la Dirección General para la Igualdad de Oportunidades dentro de los cometidos y estructura del Instituto de la Mujer, que pasa a denominarse Instituto de la Mujer y para la Igualdad de Oportunidades y depende del actual Ministerio de Sanidad, Servicios Sociales e Igualdad (desaparecido el Ministerio de Igualdad).

En el ámbito privado encontramos la Fundación Másfamilia. Desde su inicio en el año 2003, está impulsada por la Federación Española de Familias Numerosas, el Ministerio de Igualdad (Actualmente por el Ministerio de Sanidad, Servicios Sociales, e Igualdad) y un conjunto de empresas diversas. Esta fundación ha creado un modelo propio, el Modelo de Gestión efr 1000 (que da acceso a la marcar efr), para diseñar e implantar estrategias de Responsabilidad Social Corporativa en empresas y la conciliación de la vida familiar y laboral así como de igualdad de oportunidades tomando como base la legislación vigente y vinculante y la legislación colectiva (Fundación Másfamilia, n.d.). A pesar de que inicialmente la certificación de Másfamilia surge con el objeto de ser aplicada en empresas privadas, esta iniciativa se extiende a instituciones públicas (como los ayuntamientos de Salamanca, Palencia, Granada o Calviá, entre otros). Posteriormente, el modelo efr 1000 ha ido integrando otros modelos denominados suplementos sectoriales (Modelos efr: educación, 
economía social, franquicia, microentidad, global), dando visibilidad a distintas empresas clasificadas dentro de esta estructura.

2) Órgano de representación: en esta categoría observamos que los textos no exigen la existencia de una estructura orgánica que vele por el cumplimiento de la aplicación de la dimensión de género en la organización para la obtención del certificado de Igualdad en la Empresa, ni que tengan un carácter paritario. Tan sólo el punto 4 de la Orden SPI/2293/2011, de 11 de julio (España, 2011), por la que se convoca el procedimiento para la concesión del distintivo "Igualdad en la Empresa" correspondiente al año 2011, y se establecen sus bases reguladoras. La misma dicta lo siguiente: "Con carácter facultativo, las empresas solicitantes, podrán aportar un informe de la representación del personal en la empresa sobre el plan de igualdad o las políticas de igualdad en los que se apoya la solicitud" (España, 2011, p.93289).

En los análisis de publicaciones privadas, tampoco encontramos referencias que aludan a la obligatoriedad de construir órganos paritarios que garanticen la aplicación del mainstreaming de género. Igualmente, desconocemos los datos de las empresas con certificaciones de la Fundación Másfamilia en relación a la existencia y la composición de estos órganos.

3) Reconocimiento de las desigualdades de género y relaciones de poder: los textos institucionales (normativa y documentos técnicos) subrayan explícitamente el origen y mantenimiento estructural de las desigualdades de género, presentando información objetiva sobre este fenómeno. Además, llevando a cabo una caracterización de conceptos fundamentales en el marco de la aplicación del mainstreaming de género en las organizaciones. Como ejemplo, en relación a la incorporación de las mujeres al mercado laboral hacen visible la existencia de la división sexual del trabajo citando que: "el cambio en los roles tradicionales, pone al descubierto una sociedad que no está preparada para ello porque no tiene organizados los servicios públicos ni esos cuidados en horas laborales" (Ministerio de Sanidad, Asuntos Sociales e Igualdad, 2016, p.3).

No obstante, en los análisis de contenidos de algunas webs institucionales, representantes ministeriales no hacen este mismo tratamiento. En nota de prensa publicada en 2015 (en el marco de entrega de distintivos de Igualdad la empresa) en la web dice sobre la incorporación de las mujeres al empleo (España, 2015): "están produciendo notables avances, como prueban los datos de paro del mes de junio conocidos hoy: más de 94.700 personas menos en las listas de desempleo y casi un $2 \%$ de descenso del paro femenino". A continuación, nombra las empresas certificadas que la nota de prensa muestra como ejemplos a seguir de garante para la lucha de la segregación en el empleo.

A pesar de que el Ministerio de Sanidad, Servicios Sociales e Igualdad (responsable de vigilar las correcta aplicación del mainstreaming de género) avala las certificaciones privadas, a la luz del análisis de los textos de las organizaciones privadas citadas, echamos en falta los mecanismos utilizados por el Ministerio para el seguimiento y evaluación del cumplimiento de la aplicación de la transversalidad de género en estas organizaciones. Por ejemplo, encontramos en los textos de organizaciones privadas un tratamiento no acorde con los argumentos fundamentales del mainstreaming de género. Sirvan de muestra, las declaraciones que la Fundación Másfamilia distribuye a través de su blog de la conciliación donde señala: "En relación a la conciliación, la feminista coñazo/a presenta algunas singularidades: ... . Sería algo casi como sólo me interesa conciliar si consigo con ello que los tipos vivan peor" (Martínez, 2012). Otro ejemplo encontrado es cuando a través del análisis de los textos nos percatamos que el lenguaje utilizado se aleja precisamente de los pilares que sostienen la política del mainstreaming de género. Sirva de muestra el citado blog de la conciliación de Másfamilia: "Los tíos somos unicelulares. Sed pacientes. O ¿es 'pacientas'? ya no estoy seguro..." (Martínez, 2012).

4) Interseccionalidad: en esta categoría encontramos que tanto en los textos institucionales 
(documentos técnicos de ayuda y webs) como en los textos publicados por organizaciones privadas, no se profundiza en que la dimensión de género se correlaciona con otras variables que también generan desigualdades, invisibilizando el concepto de interseccionalidad. En este sentido, observamos que la mayoría de las veces se da un tratamiento bidimensional al sexo (hombre-mujer) sin promover la importancia de relacionarlo con otras dimensiones personales o culturales que pueden ocasionar discriminación múltiple y que lo denuncia la perspectiva de género. Si bien es cierto, que en algún texto se menciona que existe una relación entre la variable género y otras variables que hace que éstas se conviertan en "grupos vulnerables" (Ministerio de Sanidad, Asuntos Sociales e Igualdad, 2016, p.7), las referencias son escasas y no se utiliza ni conceptualiza el término interseccionalidad.

\section{Dimensión 2: Proyección del mainstreaming de género}

5) Marca de calidad: en los textos publicados tanto por organizaciones públicas como privadas, se lleva a cabo una promoción de la certificación de la igualdad como marca dentro de un sistema propio de gestión de calidad organizativa (a excepción de los textos normativos).

En cuanto a las instituciones públicas, El manual "Orientaciones para negociar medidas y planes de igualdad de oportunidades entre mujeres y hombres en la empresa" (Fundación Mujeres, 2008) se plantea la igualdad de oportunidades como "rentable para las empresas" porque es un "buen negocio" (p.12), dado que permite cumplir con la legislación vigente, optimizar recursos humanos, consolidar la Responsabilidad Social Corporativa y asegurar el desarrollo de los sistemas de calidad. Así, en esta guía se recomienda a las entidades "incorporar medidas de igualdad de oportunidades a través de sus propios sistemas de gestión de la calidad".

En Fundación Mujeres (2007) se destaca la creación del Comité de Normalización de Igualdad de Oportunidades entre Mujeres y Hombres en la Gestión Empresarial (Asociación Española de Normalización/Comités Técnicos de Normalización [AEN/CTN-194]) resultando la Norma UNE 194001 en Igualdad de Oportunidades que facilitará que cualquier empresa pueda certificarse en el futuro en igualdad de oportunidades, dado que "tanto las Normas ISO 9000 como el Modelo EFQM plantean como un principio básico la gestión por procesos, que permite a las organizaciones empresariales analizar las distintas actividades que lleva a cabo como un todo interrelacionado" (Fundación Mujeres, 2007, p.6).

El denominado Modelo de certificación efr 1000 de Másfamilia (Fundación Másfamilia, n.d.), para las organizaciones consideradas como familiarmente responsables, también se orienta a articular la dimensión de género en los procesos que conforman los sistemas de calidad organizativa. Los llamados prescriptores, son entidades que colaboran activamente en el diseño, implantación y evaluación del Modelo efr. Dichos prescriptores son homologados por la Fundación Másfamilia, para así convertirse en los únicos que pueden formar, implantar, auditar y certificar a las organizaciones familiarmente responsables, entre los que nos encontramos conocidas certificadoras de sistemas de calidad organizacional como la Asociación Española de Normalización y Certificación (AENOR), Lloyd's Register (LRQA), Det Norske Veritas (DNV) y Bureau Veritas (Fundación Másfamilia, n.d.).

6) Transversalidad: en esta categoría observamos que en los textos normativos y técnicos de tipo institucionales se hace explícito que aplicar el enfoque de género implica a todos los niveles y los procesos de la organización y a todo tipo de políticas y programas (Biencinto \& González, 2010). El texto del Instituto de la Mujer (2008), hace referencia a la importancia de involucrar a todos los agentes de la organización, y además "hay que determinar la fase en la que participarán, definir el tipo de participación y la forma de organizarla" (p.5). Igualmente, los textos institucionales hacen un reconocimiento explícito de que los análisis de resultados alcanzados, procesos implementados y conclusiones sirven para combatir las relaciones 
asimétricas entre los géneros (relación con mantenimiento de roles y estereotipos de género, segregación laboral de género, creación de espacios de participación para construcción de conocimiento colectivo y empoderamiento).

Sin embargo, observamos que fomentan la adopción del enfoque de género dentro de los sistemas de calidad como garante de esta transversalidad. Advertimos en textos institucionales un tratamiento de la igualdad de género como áreas independientes para su inclusión en los sistemas de calidad, como temáticas inconexas. Siendo estas: 1 . Selección y promoción de personal; 2. Medidas de conciliación de la vida laboral, familiar y personal; 3 . Acoso Sexual Laboral; 4. Normativa en materia de Igualdad; 5 . Formación con perspectiva de género en la organización; 6. Lenguaje e imágenes no sexistas en la organización; 7. Discriminación salarial (Fundación Mujeres, 2007). De ahí que no se trata de una transversalidad en los términos que supone la adaptación del mainstreaming de género. Este último subraya la importancia de no tomarla como procesos independientes, sino como un continuo.

7) Metodología: partiendo del análisis documental realizado, comprobamos que el modelo que promueve el estado español se fundamenta en un enfoque objetivo y funcionalista de la cultura organizacional. Como observamos en publicaciones como la del Instituto de la Mujer (2008), desde un punto de vista procedimental, los diagnósticos se basan en un modelo de encuesta (cuantitativo) apoyado en el estudio de fuentes documentales. El tipo de estudio que se lleva a cabo según la naturaleza de los datos es de tipo descriptivo, utilizando técnicas de recogida de información cuantitativas y cualitativas. Los análisis cualitativos se desarrollan a través de la aplicación de instrumentos como la entrevista, pero siempre que supongan una información de apoyo al análisis cuantitativo.

Otro ejemplo lo encontramos en el manual "Modelo de referencia para la elaboración de diagnósticos y diseño e implantación de planes de igualdad", que se edita con objeto de "reducir la carga administrativa en la aplicación de planes modelo de referencia, compuesto por una serie de fichas que faciliten la elaboración del diagnóstico y el diseño de un Plan de Igualdad" (Fundación Mujeres, 2011, p.3).

Este método promovido por las instituciones públicas es propio de la aplicación de sistemas de calidad organizativos orientados a la mejora continua de las empresas. Esto queda explícito en Fundación Mujeres (2007) cuando advierte que la metodología utilizada para la mejora continua por parte de las organizaciones supone una herramienta fácilmente utilizable para la introducción de objetivos en igualdad de oportunidades como estrategia hacia la calidad total "ya que es totalmente convergente con el desarrollo de Planes de Acción Positiva: ambas metodologías plantean la necesidad de empezar de un conocimiento previo de la situación a partir de distintos indicadores, del diseño o planificación de unos objetivos" (p.7).

Las organizaciones privadas responsables de la promoción de las certificaciones de igualdad, se apoyan en la metodología que promueven las instituciones públicas. El procedimiento diagnóstico que formula la Fundación Másfamilia para la certificación de empresas familiarmente responsables - Modelo efr -, se apoya en la aplicación modelos de calidad organizativa. Así, según su página web (Cembranos, 2013), consultoras especializadas ofrecen sus servicios para la aplicación de la norma efr 1000.

\section{Consideraciones Finales}

El estudio del que parte este artículo, con el ánimo de responder a la cuestión ¿de qué manera, las organizaciones públicas y privadas en España (siguiendo la iniciativa de "Distintivo de Igualdad en las empresas") están respondiendo a Ley Orgánica n 3/2007 (España, 2007) para la igualdad efectiva de mujeres y hombres?, se dio a la tarea de identificar el enfoque desde el que organizaciones públicas y privadas (con aval ministerial) responsables de la promoción de las certificaciones de igualdad en España, están respondiendo a la aplicación del mainstreaming de género. 
El estudio de lo que nos ocupa arroja que las principales organizaciones responsables de la promoción de estas certificaciones en España son: a nivel público y en sus inicios, el Ministerio de Igualdad a través del Instituto de la Mujer. Actualmente, reside sólo en el Ministerio de Sanidad, Servicios Sociales e Igualdad. Y, a nivel privado encontramos la Fundación Másfamilia que ampara otras empresas certificadas por ella, y a su vez, alberga empresas certificadoras como: AENOR, LRQA, DNV y Bureau Veritas.

Encontramos que tanto entidades públicas como privadas impulsan los distintivos (De lgualdad en las empresas y efr 1000, respectivamente) con fines mercantiles disfrazados de marcas en aras del progreso social y la equidad entre los sexos sin que realmente supongan un avance transformador. Sobre esta cuestión coincidimos con Espinosa (2014), cuando describe un escenario social donde, de manera generalizada, se asiste a una marginación de los contenidos de género en la agenda pública que comienza en la década de los ochenta a la par que la iniciativa Marca España gana centralidad hasta nuestros días.

En nuestros análisis se constata que, a pesar de que el mainstreaming de género es una estrategia que se recomienda aplicar en todos los niveles y procesos organizacionales (Berbel, 2013; Bonder, 2012; Mimbrero \& Navarro, 2011); los organismos responsables de promover las certificaciones de igualdad no hacen un tratamiento del enfoque transversal adecuado. Estas organizaciones, en su tratamiento impulsor de la aplicación del mainstreaming de género, no exigen la constitución de estructuras orgánicas que velen por su cumplimiento, ni tampoco su composición de manera paritaria. Además, son escasas las referencias para promover que la articulación del género en las organizaciones forme parte de los objetivos estratégicos. Asimismo, si bien es cierto que sobre todo en publicaciones institucionales hay un reconocimiento explícito al origen y mantenimiento de las desigualdades desde un enfoque estructural; en sus páginas webs encontramos comunicaciones más informales donde se invisibiliza. Se muestran discursos de sus representantes encuadrando las desigualdades de género en un marco interpretativo que deja al margen su origen estructural, existiendo un desequilibrio entre el diagnóstico de las realidades sociales y las soluciones planteadas. En nota de prensa sobre el acto de entrega de los distintivos de Igualdad en la Empresa en julio de 2015, la igualdad de género es tratada como marketing político, ofreciendo datos superficiales sobre la situación entre mujeres y hombres que dejan al margen las verdaderas circunstancias en torno al empleo desde una perspectiva de género (España, 2015).

Por otro lado, a pesar de que desde las instituciones públicas se hace un llamado al uso del lenguaje inclusivo y no sexista como parte de la aplicación del mainstreaming de género, las organizaciones privadas banalizan este imperativo en tanto y en cuanto, no sólo en algunas ocasiones utiliza un lenguaje machista; sino que se ridiculiza la importancia de velar por un lenguaje incluyente. Algo que comparten las publicaciones de entidades públicas y privadas es la falta de visualización del enfoque de interseccionalidad, e incluso, del propio término como tal. Numerosas aportaciones (Oliveira \& Araujo, 2014; Rodríguez, 2010; Roggeband \& Verloo, 2007; Verloo, 2006, 2015) ponen de manifiesto que la interseccionalidad es una variable que debe abordarse, sin ninguna duda, como esencial para aplicar la perspectiva de género no sólo en el contexto de las organizaciones, sino también, en cualquier otro ámbito.

Por su parte, la metodología utilizada tanto por organizaciones públicas como privadas, no están acordes con las propuestas feministas que aluden a una metodología más cualitativa (Ligero, Espinosa, Mormeneo, \& Bustelo, 2014). Analizando distintas ediciones hemos comprobado que la estructura evaluativa que se impulsa se inspira en un modelo de análisis enmarcado en una visión objetivista y funcional y que va asociado a la calidad organizativa (que se vincula al modelo económico neoliberal). Cuestión que entronca con el debate actual (Addabbo, Rodriguez Modroño, \& Gálvez Muñoz, 
2013; Blyth, 2013; Gálvez, 2013) - surgido en el marco de la globalización y crisis financiera -, sobre el impacto desigual que las políticas neoliberales y el "austericidio" ejercen sobre mujeres y hombres, y de cómo estas prácticas y consecuencias se trasladan al ámbito organizativo.

Todo lo anteriormente dicho pone sobre la mesa el riesgo de que la adopción del enfoque de género en las organizaciones tienda a ponerse al servicio de criterios mercantilistas. A pesar de que reconocemos que estas certificaciones pueden visibilizar entidades (de capital público y privado) que incorporan la dimensión de género. Además, promociona la incorporación de buenas prácticas desde una perspectiva de igualdad entre mujeres y hombres en las organizaciones. No obstante, de los textos analizados se desprende una posible promoción de certificaciones que pueden contribuir a impulsar el interés mercantil de empresas, más que ser aseguradoras de la adopción de la filosofía del mainstreaming de género. Un modelo mercantilista, elitista y competitivo que favorece a los sistemas que promueven incoherentemente valores opuestos a los que proclama: la desigualdad, la discriminación o el materialismo.

Así, sobre la base de estos resultados, reconocemos la importancia científica de visibilizar si la adopción de un enfoque de género en las organizaciones corre el riesgo de tecnocratizarse desde un punto de vista político. Además, si este hecho está promoviendo la aplicación de herramientas políticas como las certificaciones de igualdad, desde una perspectiva que no contribuye a combatir las diferencias de género. Igualmente, si está favoreciendo que las cuestiones de género sean tratadas desde un enfoque mercantil, como marca empresarial. Por ello, reivindicamos la aplicación de modelos culturales y participativos en los que se reconozcan y visibilicen las verdaderas necesidades $y$ motivaciones de mujeres y hombres que conforman las organizaciones con el objeto de intervenir sobre una realidad que, por intereses patriarcales y del mercado, puedan pretender que permanezcan ocultas. A la par, alertamos sobre la necesidad de formular profundas reflexiones científicas, sociales y políticas acerca del impacto que están ejerciendo estas prácticas organizativas (como estrategias de monetización de las ideas neoliberales) en el marco europeo y también fuera de Europa. Y así, poder revelar si se trata de una tendencia que se extiende en varios países y emprender soluciones al respecto.

Las limitaciones de este estudio residen en el no análisis de todas las publicaciones existentes en el marco de la igualdad certificada en España. Por ello se sugiere en futuras investigaciones incrementar el número de textos a analizar. Además, que una vez habiendo hecho esto, se amplíe el estudio en otros ámbitos territoriales añadiendo al diseño de la investigación el que sea longitudinal para ver si ocurren transformaciones durante el tiempo.

\section{Contributors}

C. MIMBRERO MALLADO fue la responsable de la concepción y diseño, análisis de datos e interpretación así como revisión y aprobación de la versión final del artículo. L.M. CANTERA ESPINOSA participó en el análisis de datos e interpretación así como revisión y aprobación de la versión final del artículo. J.P. SILVA contribuyó en la revisión y la aprobación de la versión final del artículo.

\section{References}

Addabbo, T., Rodríguez Modroño, P., \& Gálvez Muñoz, L. (2013). Gender and the great recession: Changes in labour supply in Spain. DEMB Working Paper Series, 10, 1-28. Retrieved October, 5, 2014, from http://merlino.unimo.it/campusone/web_dep/ wpdemb/0010.pdf

Berbel, S. (2013). Directivas y empresarias: Mujeres rompiendo el techo de cristal. Barcelona: Aresta.

Berg, B. L. (1989). Qualitative research methods for the social sciences. Massachusetts: Allyn and Bacon.

Biencinto, N., \& González, Á. (2010). La Transversalidad de género: Métodos y técnicas. Sevilla: Instituto Andaluz de la Mujer.

Blyth, M. (2013). Austerity. The history of a dangerous Idea. Oxford: Oxford University Press. 
Bonder, G. (2012). Fundamentos y orientaciones para la integración del enfoque de género en políticas, programas y proyectos. In Bonder, G. Sinergias. Cuadernos del Área Género, Sociedad y Políticas. Argentina: Facultad Latinoamericana de Ciencias Sociales.

Brasil. Ministério da Saúde. (1996). Resolução n 196, de 10 de outubro de 1996. Aprova as diretrizes e normas regulamentadoras de pesquisas envolvendo seres humanos. Diário Oficial da União, vol. 134, n 201, out.1996, Seção 1, pp.20182-20186.

Brown, J. (2007). Mujeres y ciudadanía: De la diferencia sexual como diferencia política. Kairos: Revista de Temas Sociales, 19. Recuperado en marzo 5, 2014, de https:// dialnet.unirioja.es/descarga/articulo/2509380.pdf

Bustelo, M., \& Lombardo, E. (2005). Mainstreaming de género y análisis de los diferentes 'marcos interpretativos' de las políticas de igualdad en Europa: El proyecto MAGEEQ. Aequalitas: Revista Jurídica de Igualdad de Oportunidades entre Mujeres y Hombres, 17, 15-26.

Bustelo, M., \& Lombardo, E. (2006). Los 'marcos interpretativos' de las políticas de igualdad en Europa: conciliación, violencia y desigualdad de género en la política. Revista Española de Ciencia Política, 14, 117-140.

Cembranos, E. (2013). ¿Cómo implantar la norma efr 1000-1? Recuperado en enero 10, 2016, de http:// www.erreese.com/gestion-de-las-personas/comoimplantar-la-norma-efr-1000-1/

Díaz, C., \& Dema, S. (2013). Sociología y género. Madrid: Tecnos.

España. (2007). Ley Orgánica 3/2007, de 22 de marzo, para la igualdad efectiva de mujeres y hombres. Boletín Oficial del Estado, $n^{\circ}$ 71, de 23 de marzo de 2007, pp.12611-12645.

España. (2014). Ley 15/2014, de 16 de septiembre, de racionalización del Sector Público y otras medidas de reforma administrativa. Boletín Oficial del Estado, $n^{\circ}$ 226, de 17 de septiembre de 2014, pp.1-47.

España. Ministerio de Igualdad. (2009a). Orden IGD/3195/2009, de 12 de noviembre, por la que se aprueba el logotipo y representación grafica del distintivo "Igualdad en la empresa". Boletín Oficial del Estado $n^{\circ} 286$, de 27 de noviembre de 2009, Sección III, pp.100852-100853.

España. Ministerio de la Presidencia. (2009b). Real Decreto 1615/2009, de 26 de octubre, por el que se regula la concesión y utilización del distintivo "Igualdad en la Empresa". Boletín Oficial del Estado, n² 265, Sección I, pp.91703-91712.

España. Ministerio de Sanidad, Servicios Sociales e Igualdad. (2011). Orden SPI/2293/2011, de 11 de julio, por la que se convoca el procedimiento para la concesión del distintivo "Igualdad en la Empresa" correspondiente al año 2011, y se establecen sus bases reguladoras. Boletín Oficial del Estado, $n^{\circ} 201$, de 22 de agosto de 2011, Sección III, pp.93286-93312.

España. Ministerio de Sanidad, Servicios Sociales e Igualdad. (2015). Susana Camarero apuesta por la colaboración mutua y la unión de voluntades para avanzar hacia la "igualdad real" de oportunidades. Recuperado el enero 16, 2015, de http://www.msssi. gob.es/gabinete/notasPrensa. do?id=3707

Espinosa, J. (2014). La igualdad de género en la cooperación española y en la iniciativa Marca España. Abordajes e inconsistencias en tiempos de crisis. Revista de Investigaciones Feministas, 5, 259-288. https://doi. org/10.5209/rev_INFE.2014.v5.48135

Fundación Másfamilia. (n.d.). Fundación Másfamilia. Recuperado el octubre 5, 2014, de http://www. Másfamilia.org

Fundación Másfamilia. (n.d.). Prescriptores efr. Recuperado el enero 10, 2016, de http://www.Másfamilia.org/ empresa?listid $=20$

Fundación Mujeres. (2007). Manual de procedimientos para la incorporación de la igualdad en la gestión empresarial. Principado de Asturias: Autor.

Fundación Mujeres. (2008). Orientaciones para negociar medidas y planes de igualdad de oportunidades entre mujeres y hombres en la empresa. Madrid: Instituto de la Mujer.

Fundación Mujeres. (2011). Modelo de referencia para la elaboración de diagnósticos y diseño e implantación de planes de igualdad. Madrid: Instituto de la Mujer.

Gálvez, L. (2013). Una lectura feminista del austericidio. Revista de Economía Crítica, 15, 80-110. Recuperado en enero 10, 2015, de http://revistaeconomiacritica. org/sites/default/files/revistas/n15/Crisis02_linagalvez. pdf

Instituto de la Mujer. (1999). Mainstreaming de género: Marco conceptual, metodología y presentación de "buenas prácticas": Informe final de las actividades del Grupo de especialistas en "mainstreaming". Madrid: Autor.

Instituto de la Mujer. (2008). Manual de para elaborar un plan de igualdad en la empresa. Aspectos básicos. Recuperado en diciembre 5, 2014, de https://www. msssi.gob.es/ssi/igualdadOportunidades/docs/Manual_ para_elaborar_un_Plan_lgualdad_en_la_empresa.pdf

Instituto de la Mujer y la Unidad Administradora del Fondo Social Europeo. (2013). Protocolo para la implantación de un proceso de mainstreaming de género. Recuperado en marzo 7, 2016, de http://www.inmujer. gob.es/areasTematicas/redPoliticas/actividadesRed/ docs/SesionPlenariaAviles/09_Protocolo_MG_def.pdf 
Ligero, J. A., Espinosa, J., Mormeneo, C., \& Bustelo, M. (2014). Cómo hacer una evaluación sensible al género y al enfoque basado en derechos humanos para el desarrollo. Revista Vasca de Sociología y Ciencia Política, 57-58, 3032-3039.

Lombardo, E. (2005). Integrating or setting the agenda? Gender mainstreaming in the European constitutionmaking process. Social Politics: International Studies in Gender, State and Society, 12(3), 412-432. https:// doi.org/10.1093/sp/jxi022

Martínez, L. M., Biglia, B., Luxán, M., Fernández Bessa, C., Azpiazu Carballo, J., \& Bonet Martí, J. (2014). Experiencias de investigación feminista: Propuestas y reflexiones metodológicas. Athenea Digital, 14(4), 3-16. https://doi.org/10.5565/rev/athenea.1513

Martínez, R. (2012, Mayo 16). ¿Quién es? La feminista coñazo [Web blog post]. Recuperado en octubre 5, 2014, de http://conciliatorvsdarkworker.blogspot.com. es/ 2012/05/quien-es-quien-la-feminista-conazo.html

Mimbrero, C. (2014). Nuevo modelo diagnóstico de la cultura de género en la administración local y en el tercer sector de acción local (Tesis doctoral no publicada). Universidad de Sevilla, España. Recuperado en febrero 14, 2016, de http://fondosdigitales.us.es/ tesis/tesis/2712/nuevo-modelo-diagnostico-de-lacultura-de-genero-en-la-administracion-local-y-en-eltercer-sector-de-accion-social/

Mimbrero, C., \& Navarro, P. (2011). Género y exclusión social. Guía para la incorporación del enfoque de género en programas y proyectos. Sevilla: Federación Enlace e Instituto Andaluz de la Mujer.

Ministerio de Sanidad, Asuntos Sociales e Igualdad. (2016). Herramienta de apoyo $n^{\circ}$ 6: Conciliación de la vida laboral. Madrid: Autor. Recuperado en febrero 10, 2016, de http://www.igualdadenlaempresa.es/ recursos/herramientas/docs/herramienta_Apoyo_6_ conciliacion_vida_laboral.pdf

Olabuénaga, J. I. (2012). Metodología de la investigación cualitativa (5 ed.). Bilbao: Universidad de Deusto.

Oliveira, E., \& Araujo M. (2014). O Teatro Fórum como dispositivo de discussão da violência contra a mulher. Estudos de Psicologia (Campinas), 31(2), 257-267. https://doi.org/10.1590/0103-166X2014000200011
Pla, M. (1999). El rigor en la investigación cualitativa. Atención Primaria, 24(5), 295-300.

Reverter, S. (2011). La dialéctica feminista de la ciudadanía. Athenea Digital, 11(3), 121-136. https:// doi.org/10.5565/rev/athenead/v11n3.758

Rodríguez, B. (2010). Hacia un Estado post-patriarcal. Feminismo y ciudadanía. Revista de Estudios Políticos, 149, 87-122. Recuperado en febrero 10, 2016, de http://recyt.fecyt.es/index.php/RevEsPol/article/ view/44319/25936

Roggeband, C., \& Verloo, M. (2007). Dutch women are liberated, migrant women are a problem: The evolution of policy frames on gender and migration in the Netherlands, 1995 - 2005. Social Policy \& Administration, 41(3), 271-288. https://doi. org/10.1111/j.1467-9515.2007.00552.x

Verloo, M. (2001). Another velvet revolution? Gender mainstreaming and the politics of implementation. Viena: IWM Working Papers.

Verloo, M. (2005). Displacement and empowerment: Reflections on the Council of Europe approach to gender mainstreaming and gender equality. Social Politics, 12(3), 344-366. https://doi.org/10.1093/sp/ jxi019

Verloo, M. (2006). Multiple inequalities, intersectionality and the European Union. European Journal of Women's Studies, 13(3), 211-229. https://doi. org/10.1177/1350506806065753

Verloo, M. (2015). Intersectional and cross-movement politics and policies: Reflections on current practices and debates. Intersectionality: Theorizing Power, Empowering Theory, 38(4), 893-915. https:// doi. org/10.1086/669572

Verloo, M., \& Roggeband, C. (1996). Gender impact assessment: The development of a new instrument in the Netherlands. Impact Assessment, 14(1), 3-20.

https://doi.org/10.1080/07349165.1996.9725883

Recibido: Junio 15, 2016

Versión final: Noviembre 22, 2016

Aprobado: Noviembre 24, 2016 